\title{
Cancer Signature Investigation: ERBB2 (HER2)-Activating Mutation and Amplification-Positive Breast Carcinoma Mimicking Lung Primary
}

\author{
Jennifer Shih, MD, MS ; Babar Bashir, MD ; Karen S. Gustafson, MD, PhDa; Mark Andrake, PhD; \\ Roland L. Dunbrack, PhDa; Lori J. Goldstein, MDª and Yanis Boumber, MD, PhDa,c
}

\begin{abstract}
Next-generation sequencing of primary and metachronous metastatic cancer lesions may impact patient care. We present a case of relapsed metastatic breast cancer with a dominant pulmonary lesion originally identified as lung adenocarcinoma. A 72-year-old, never-smoker woman with a protracted cough was found to have a large lung mass and regional lymphadenopathy on a chest CT. Lung mass biopsy showed adenocarcinoma with focal TTF-1 (thyroid transcription factor 1) positivity, favoring a lung primary. In addition to stereotactic brain radiation for cerebral metastases, she was started on carboplatin/pemetrexed. As part of the workup, the tumor was analyzed by a 50-gene targeted mutation panel, which detected 3 somatic mutations: ERBB2 (HER2) D769H activating missense mutation, TP53 Y126 inactivating truncating mutation, and SMARCB1 R374Q missense mutation. Of note, the patient had a history of stage IIA triple-negative grade 3 invasive ductal carcinoma of the left breast 1.5 years ago and received neoadjuvant chemotherapy and adjuvant radiation, and underwent a lumpectomy. Further analysis of her primary breast tumor showed a mutational profile identical to that of the lung tumor. Fluorescence in situ hybridization revealed HER2 amplification in the lung tumor, with a HER2/CEP17 ratio of 3.9. The patient was diagnosed with recurrent HER2-positive metastatic breast carcinoma with a coexisting ERBB2 (HER2) activating mutation. Chemotherapy was adjusted to include dual HER2-targeted therapy containing trastuzumab and pertuzumab, resulting in an ongoing partial response. This case demonstrates that a unique genetic mutational profile can clarify whether a tumor represents a metastatic lesion or new malignancy when conventional morphological and immunohistochemical methods are indeterminate, and can directly impact treatment decisions. (J Natl Compr Canc Netw 2015;13:947-952)
\end{abstract}

Cancer is a disease of genomic alterations and, based on the NCCN Clinical Practice Guidelines in Oncology (NCCN Guidelines), the use of therapeutic agents that target specific genomic alterations is standard of care for several tumor types, including lung and breast carcinomas. ${ }^{1,2}$ First-line treatment of lung adenocarcinomas that have a sensitizing EGFR mutation or ALK rearrangement includes the use of a tyrosine kinase inhibitor, such as erlotinib or crizotinib, whereas ceritinib is currently approved for ALK-positive patients in the second-line setting. ${ }^{2}$ Similarly, first-line treatment of breast carcinomas with amplification of ERBB2

From ${ }^{\text {aFox }}$ Chase Cancer Center, Temple University Heath System,

Philadelphia; ${ }^{b}$ Abington Memorial Hospital, Abington; and 'Molecular Therapeutics Research Program, Fox Chase Cancer Center, Philadelphia, Pennsylvania.

Submitted December 10, 2014; accepted for publication April 29, 2015.

Dr. Boumber has disclosed that he receives research support from Synta Pharmaceuticals Corp for product development; is on the advisory board for Bristol-Myers Squibb Company and Clovis Oncology; and is on the
(HER2) includes trastuzumab and pertuzumab, whereas in the second-line setting, ado-trastuzumab emtansine and lapatinib have been approved (NCCN Guidelines for Breast Cancer). ${ }^{1}$ Given the central role of targeted agents in the management of patients with tumors harboring these types of changes, testing for genomic alterations is recommended as part of the workup in the NCCN Guidelines for several tumor types. Importantly, as the number of clinically relevant genes and targeted therapies for different tumor types expand, testing using a multiplex, high-throughput next-generation sequencing (NGS) platform offers a method for analyzing the

speakers' bureau for Synta Pharmaceuticals Corp. The remaining authors have disclosed that they have no financial interests, arrangements, affiliations, or commercial interests with the manufacturers of any products discussed in this article or their competitors.

Correspondence: Jennifer Shih, MD, MS, Fox Chase Cancer Center, 333 Cottman Avenue, Philadelphia, PA 19111.

E-mail: jennifer.shih@fccc.edu

Yanis Boumber, MD, PhD, Fox Chase Cancer Center, 333 Cottman Avenue, Philadelphia, PA 19111. E-mail: YBoumber@salud.unm.edu 
Shih et al

mutation status of multiple cancer-related genes, thus providing a genetic mutational profile of the tumor. This article describes a case in which the unique cancer signature (genetic mutational profile) of a tumor not only established the correct diagnosis, but also dramatically changed the course of treatment.

\section{Case Presentation}

The patient is a 72-year-old Caucasian female without a family history of cancer but with a personal history of breast cancer. In 2012, at 70 years of age, she was found to have a left breast abnormality on a screening mammogram, and the result of a biopsy performed under ultrasound showed grade 3 invasive ductal carcinoma (clinical stage IIA, T2NOMO) that was estrogen receptor (ER)-negative, progesterone receptor (PR)-negative, and HER2 (ie, ERBB2)negative (triple-negative breast cancer [TNBC]; HER2 immunohistochemical stain was 0). She received neoadjuvant dose-dense doxorubicin/cyclophosphamide for 4 cycles and dose-dense paclitaxel for 1 cycle. The latter was stopped early because of hypersensitivity pneumonitis. She underwent left lumpectomy with sentinel lymph node biopsy, and her pathologic staging was ypT2NOMO, stage IIA. She then completed adjuvant radiation after surgery. She continued on an every-3-month clinical surveillance until March 2014, when she developed a protracted cough that led to an emergency room visit.

Chest radiograph and contrast CT showed a large right upper lobe lung mass with mediastinal, hilar, and supraclavicular lymphadenopathy (Figure 1A). Results of a fine-needle aspiration biopsy of the lung mass showed poorly differentiated adenocarcinoma that was positive for $\mathrm{CK} 7$, focally positive for TTF-1, and negative for p63 and ER (Figure 2). Based on the morphology and immunohistochemical profile, a lung primary was favored. A staging PET/CT confirmed the CT findings of at least stage IIIb disease. A brain MRI unfortunately showed 2 small cerebral metastases in the right and left frontal lobes, and she was diagnosed as stage IV lung cancer.

The patient had never been a smoker but grew up exposed to second-hand smoke.

With a good clinical performance status (ECOG 1) and minimal clinical symptoms, she received stereotactic brain radiation to the 2 frontal lesions before starting palliative chemotherapy with carbopla- tin/pemetrexed for 2 cycles with a partial response (Figure 1B). She tolerated chemotherapy very well, and her cough completely resolved.

As part of the workup for presumed stage IV lung cancer, NGS-based mutational analysis was performed on the tumor from the lung using the Ion Torrent Personal Genome Machine sequencer to identify somatic mutations in targeted regions of 50 cancer-related genes, including EGFR and other lung cancer-relevant genes. Three somatic mutations were detected by NGS: ERBB2 (HER2) D769H activating missense mutation, TP53 Y126* inactivating truncating mutation, and SMARCB1 (SWI/SNF-related matrix-associated actin-dependent regulator of chromatin subfamily $B$ member 1 ) R374Q missense mutation. Because of the presence of the ERBB2 point mutation, NGS-based mutational analysis was requested on the original invasive ductal carcinoma of the breast, which revealed the same 3 somatic mutations in ERBB2, TP53, and SMARCB1. These findings provided strong genetic evidence that the tumor in the lung was actually a metastasis from the breast primary, because the 2 tumors shared the identical genetic mutational profile. Immunohistochemistry (IHC) for HER2 expression performed on the lung tumor, according to guideline recommendations, ${ }^{3,4}$ was positive $(3+)$, and fluorescence in situ hybridization (FISH) for HER2 showed HER2 amplification with a HER2/CEP17 ratio of 3.9. Her chemotherapy was changed to docetaxel/ trastuzumab/pertuzumab to treat ER/PR-negative and HER2-positive metastatic breast cancer, and was subsequently switched to carboplatin/trastuzumab/ pertuzumab because of a hypersensitivity reaction to docetaxel. To date, she has experienced an ongoing partial response.

\section{Discussion}

Recurrence of TNBC with residual disease after neoadjuvant chemotherapy is common and predictable. The most common sites for breast recurrence are bones, liver, and lung, and TNBC has more propensity to metastasize to viscera and brain. ${ }^{1,5-7}$ In light of this, the present case illustrates that a more thorough evaluation of patients who are nonsmokers with a history of TNBC and presumed lung cancer is needed to ensure the accuracy of the diagnosis. The patient presented had a prior history of stage IIA left 

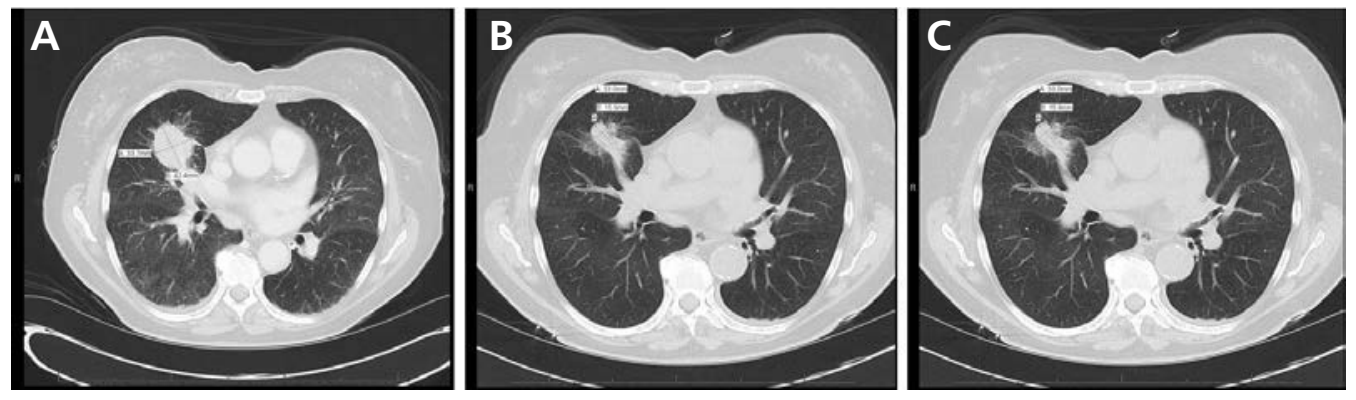

Figure $1 \mathrm{CT}$ images of the chest and abdomen after oral and intravenous contrast administration at (A) baseline, (B) after 2 cycles of carboplatin and pemetrexed, and (C) after HER2-directed therapy.

TNBC in 2012, and developed what appeared to be primary lung adenocarcinoma 1.5 years after her initial breast cancer diagnosis with brain metastases. The clinical presentation mimicked lung cancer, with a large solitary right upper lobe mass with mediastinal, hilar, and supraclavicular lymphadenopathy. The fine-needle aspiration biopsy showed adenocarcinoma that was positive for CK7, focally positive for TTF-1, and negative for p63 and ER, which favored a lung primary. Genetic mutational profiling of the lung tumor revealed 3 distinct somatic mutations in the ERBB2 (HER2), TP53, and SMARCB1 genes. Although HER2 mutations have been reported in breast cancer, HER2 driver mutations have also been reported in approximately 1.7\% (65 of 3800) of lung adenocarcinomas, ${ }^{8,9}$ and there are ongoing clinical trials for HER2-targeted therapy in lung cancer (ClinicalTrials.gov identifier: NCT01670877).

This patient received treatment for lung adenocarcinoma until it was determined that the tumor from the lung had the identical genetic mutational profile as the original breast carcinoma, which proved that she in fact had metastatic breast cancer to the lung, lymph nodes, and brain. Although TNBC can certainly recur and metastasize in this pattern within the first 3 to 5 years, ${ }^{10}$ it is relatively less common for them to mimic primary lung adenocarcinomas.

Primary lung adenocarcinoma can frequently be differentiated from a metastasis to the lung by using a panel of immunohistochemical stains, including TTF-1, CK7, CK20, and others, depending on the clinical history. ${ }^{11,12}$ However, the distinction between primary lung adenocarcinoma and metastatic TNBC can be very challenging because, by definition, the ER and PR breast biomarkers are negative. In addition, TTF-1 positivity has been reported in $2.4 \%$ of breast cancer specimens. ${ }^{13}$ More recently, additional tests have been suggested and occasionally used clinically to clarify the diagnosis of unknown primary tumors, such as CancerTYPE ID or other RNA - or miRNA-based profiling tests, or NGS approaches. ${ }^{14-16}$ Nevertheless, these are not yet widely accepted and may require a substantial amount of tissue for testing, and therefore their use is largely limited to academic centers. Furthermore, the NCCN Guidelines for Occult Primary ${ }^{17}$ do not yet recommend gene profiling or NGS as standard management (to view the most recent version of these guidelines, visit NCCN.org). The 50-gene panel used at our institution to help guide targeted therapy or trial selection for patients with solid tumors, such as those with lung adenocarcinoma, is a laboratorydeveloped test based on the Ion AmpliSeq Cancer Hotspot Panel v2, which includes targeted regions of 50 cancer-relevant genes, including EGFR, KRAS, $B R A F$, and ERBB2 (HER2). Indeed, the timing

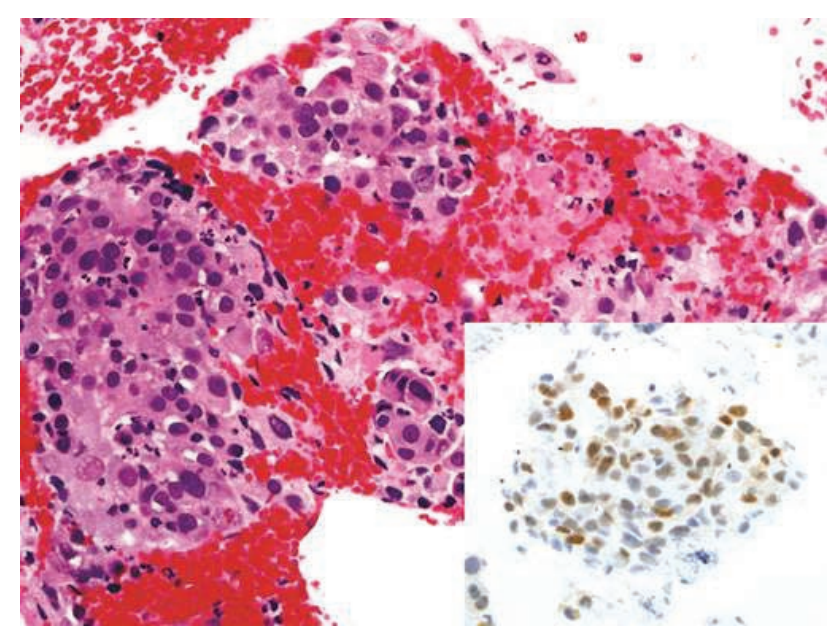

Figure 2 Histopathology of adenocarcinoma in the lung. Fine-needle aspiration biopsy of the tumor in the lung shows adenocarcinoma (formalin-fixed paraffin-embedded cell block, hematoxylin and eosin, original magnification $x 400)$. The inset shows focal nuclear staining of tumor cells with TTF-1 (original magnification $\times 400$ ). 
and exact genes tested are an area of active investigation and remain experimental. Furthermore, therapeutic decisions based on genetic abnormalities identified by NGS are not yet proven to have superior outcomes compared with standard clinical parameters guiding these decisions. Several recent publications suggest that targeted therapy in breast and other cancers based on NGS profiling could lead to a progression-free and overall survival benefit. ${ }^{18-20}$ Therefore, NGS could be used clinically in the near future if validated by prospective randomized studies (ClinicalTrials.gov identifier: NCT01670877). ${ }^{21}$ Interestingly, this patient not only carried the ERBB2 (HER2) D769H mutation, but the HER2 gene was also amplified ( $3+$ by IHC and 3.9 by FISH) in the recurrent tumor. Repeat testing of the primary breast tumor by HER2 IHC showed the tumor was HER2negative $(\mathrm{IHC} 1+)$ despite the presence of the HER2 driver mutation. These discrepancies in HER2 status between the primary breast tumor and recurrent metastatic tumor may likely reflect clonal heterogeneity and selection. The recurrent HER2-positive clone was likely present at initial diagnosis but was probably selected for metastatic spread, and recurred as the dominant clone. This clonal heterogeneity and clonal evolution is well described in primary versus metastatic lesions, ${ }^{22,23}$ and the clonal mutations that passed on from the primary to metastatic disease in this case underscores the clinical relevance of this phenomenon.

Approximately $5 \%$ to $10 \%$ of patients have HER2 status changes at distant metastasis. ${ }^{24}$ Analysis of 235 patients with metastatic breast cancer found discordance in one or more receptors between the primary tumor and metastatic breast cancer in $42 \%$ of cases. ER, PR, and HER2 status change was found to be $17 \%, 29 \%$, and $4 \%$, respectively. ${ }^{25}$ Liedtke et $\mathrm{al}^{26}$ reported ER, PR, and HER2 receptor discordance between primary and recurrent breast cancers in $18.4 \%, 40.3 \%$, and $13.6 \%$ of cases, respectively. Therefore, a biopsy is always indicated at the diagnosis of metastatic breast cancer, whether distant or locoregional. Whether routine ERBB2 (HER2) and/or gene panel NGS interrogation would become standard in clinical practice in breast oncology remains to be seen. Currently, we believe that such testing may be limited to cases in which the diagnosis is unclear, or perhaps to help select patients with metastatic breast cancer who are suitable for targeted therapy trials, because not all mutations are presently actionable. Nevertheless, with the dramatic reduction of sequencing costs to approximately $\$ 1000,{ }^{27}$ and ongoing expansion of targeted treatment options, one could foresee expansion of the use of such testing if ongoing prospective trials demonstrate utility. With an evolving list of new targets and drugs in testing, NGS technology could rapidly become a valuable resource in the search for biomarkers for newly approved targeted drugs in the near future. Indeed, this case is suggestive of the potential benefit of the NGS approach in both clarifying the diagnosis and therapeutic decisions. Currently, many prospective trials have been conducted or are underway using NGS as an approach for therapy allocation, including the NCI-MATCH (Molecular Analysis for Therapy Choice) and LungMAP (Lung Cancer Master Protocol) in lung, neratinib for HER2 mutant breast cancer (ClinicalTrials. gov identifier: NCT01670877), and others. ${ }^{21}$ Data from these and other trials may help to determine if the NGS approach will become routine practice in the near future, or will continue to be used for select patients only.

A recent paper by Bose et $\mathrm{al}^{9}$ was the first to describe a $1.7 \%$ prevalence of HER2 activating mutations in breast cancer, of which most $(80 \%)$ were HER2-negative, 12\% were HER2-positive, and the remaining $8 \%$ were HER2 equivocal. All of these mutations were sensitive to the irreversible kinase inhibitor neratinib, and either were relatively less sensitive or were resistant to lapatinib. Interestingly, of the 17 mutations described, the only one that was also unequivocally HER2-positive was the D769H mutation. ${ }^{9,28}$ This mutation was also reported in one patient with squamous cell lung cancer, although the prevalence of this HER2 mutation in these cancers is probably extremely rare. ${ }^{29}$

The ERBB2 D769H mutation is a missense mutation within the protein kinase domain of the HER2 protein, which has been shown to be an activating mutation, ${ }^{9}$ because ERBB2 $\mathrm{D} 769 \mathrm{H}-\mathrm{ex}$ pressing breast cancer cells increase the in vitro kinase activity of HER 2. ${ }^{9}$ The cell line harboring the ERBB2 D769H mutation was sensitive to neratinib, trastuzumab, and, although less so, to lapatinib. ${ }^{9}$ The D769H mutation results in substitution of asparagine to histidine at codon 769 in the alpha-helix within the kinase domain (Figure 3A), which is important 
in the binding of ATP with ERBB2. ${ }^{29}$ By examining the HER2 structure (PDB code 3PPO, and derived heterodimer models), others have noted that either the loss of the acidic side chain or the addition of an aromatic ring at amino acid 769 must activate the HER2 tyrosine kinase, due to HER2-HER3 kinase domain dimer interactions, involving residues HER2 Y772 and HER3 residues I919 and M923.9,30 It was therefore postulated that the D769H/Y mutations may increase hydrophobic contacts across this interface, and increase HER2 heterodimerization. ${ }^{9}$ Alternatively, we note that a $\mathrm{D} 769 \mathrm{H}$ change could have activating effects within the HER2 monomer by added hydrogen bonding with its own activation loop (see Figure 3A). The analogous activating mutation in EGFR $(\mathrm{D} 761 \mathrm{H})$ examined in the activatedstate crystal structure (Protein Data Bank [PDB] code $1 \mathrm{M} 17$, altered numbering $\mathrm{D} 737 \mathrm{H}$ ) would result in increased interactions with residues in the kinase activation loop. ${ }^{31}$ Finally, HER2 D769 is a highly conserved residue, invariant in all species down to the zebrafish homolog (Figure 3B), confirming that a drastic $\mathrm{D} 769 \mathrm{H}$ change is predicted to be deleterious to regulated kinase function.

\section{Conclusions}

This case report illustrates a treatment benefit provided by application of NGS technology for a patient with questionable primary lung cancer based on pathologic and immunohistochemical evaluation. Matching the genetic profile of the TNBC diagnosed 1.5 years before the new tumor in the lung allowed reclassification of her lung adenocarcinoma into a HER2-mutant, HER2-positive recurrent breast cancer. Because of the presence of HER2 positivity of the recurrent breast tumor, and the evidence that the HER2 mutation is activating, the patient's chemotherapy was then appropriately adjusted to include dual HER2-targeted therapy with docetaxel, trastuzumab, and pertuzumab, as in the CLEOPATRA trial. ${ }^{32}$ During her second cycle, she could not tolerate docetaxel because of a hypersensitivity reaction, and her therapy was changed to carboplatin, trastuzumab, and pertuzumab, and she experienced an ongoing partial response.

Genetic mutational profiling using NGS, although not yet standard in patients with breast cancer or in patients with unknown primary tumors, may be extremely valuable in not only finding targetable genetic mutations but also potentially yielding relevant diagnostic information when a unique genetic mutational profile is detected. A prospective, multiinstitutional clinical trial of neratinib in metastatic HER2 non-amplified but HER2 mutant breast cancer (ClinicalTrials.gov identifier: NCT01670877) is currently ongoing. We feel that the data presented

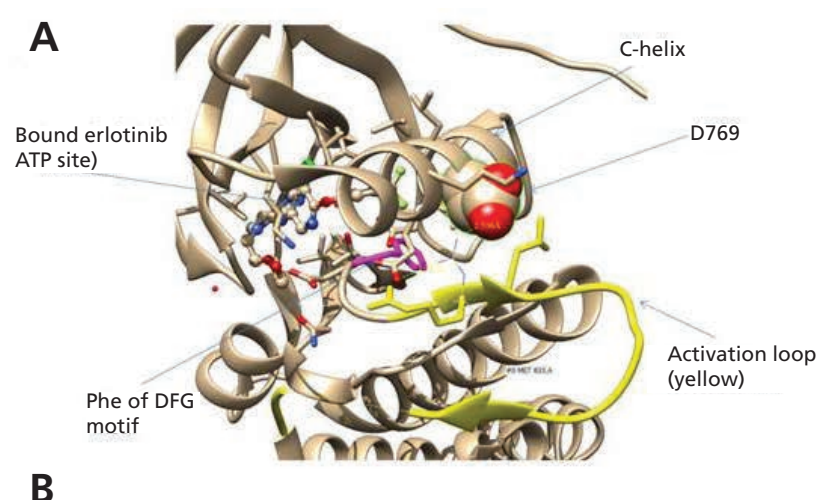

\section{$\underline{\text { Species }}$}

Human
Chimp
Gorilla
Orangutan
Rhesus
Baboon
Marmoset
Mouse Lemur
Mouse
Rat
Kangaroo Rat
Guinea Pig
Squirrel
Rabbit
Pika
Cow
Horse
Cat
Dog
Microbat
Megabat
Elephant
Rock Hyrax
Armadillo
Opossum
Wallaby
Platypus
Chicken
Lizard
X tropicalis
Tetraodon
Fugu
Stickleback
Medaka
Zebrafish
Lamprey

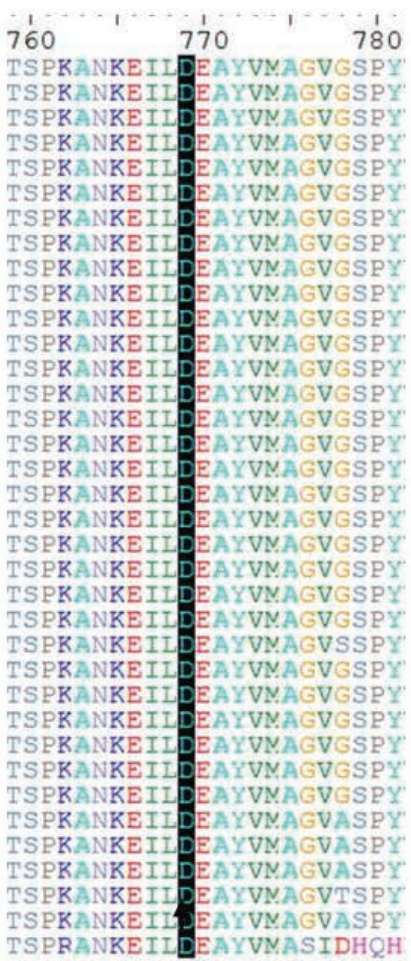

Figure 3 HER2 structure and D769H location and conservation. (A) HER2 structure (Protein Data Base [PDB] code 3PP0) is shown focused on the region of D769 shown as spheres. The bound erlotinib inhibitor is shown as ball-and-stick, and the phenylalanine of the DFG motif is shown as magenta sticks. The activation loop is shown in yellow, and the figure is prepared with the UCSF Chimera Software. ${ }^{33}$ (B) Conservation of D769 region of HER2 proteins where the species are listed on the left, and D769 analogous residues have dark highlighting. The alignment was produced with MEGA6 software ${ }^{34}$ and displayed with BioEDIT. ${ }^{35}$ 
herein further support the efforts to pursue detailed molecular characterization in select patients with cancer, especially in those with a history of breast cancer, regardless of ER, PR, or HER2 status; in patients with questionable primary tumor site; or in patients with a prior diagnosis of any other cancer type. Clinically relevant mandatory biomarker testing (ER, PR, HER FISH/IHC for patients with prior breast cancer history, with the addition of NGS for select patients) reflects a personalized medicine approach, which allows one to find new therapeutic targets and identify the subset of patients who may derive benefit from targeted therapies that can dramatically impact their care.

\section{References}

1. Gradishar WJ, Anderson BO, Blair SL, et al. Breast cancer, version 3.2014. J Natl Compr Canc Netw 2014;12:542-590.

2. Ettinger DS, Wood DE, Akerley W, et al. Non-small cell lung cancer, version 1.2015. J Natl Compr Canc Netw 2014;12:1738-1761.

3. Wolff AC, Hammond ME, Schwartz JN, et al. American Society of Clinical Oncology/College of American Pathologists guideline recommendations for human epidermal growth factor receptor 2 testing in breast cancer. J Clin Oncol 2007;25:118-145.

4. Wolff AC, Hammond ME, Hicks DG, et al. Recommendations for human epidermal growth factor receptor 2 testing in breast cancer: American Society of Clinical Oncology/College of American Pathologists clinical practice guideline update. J Clin Oncol 2013;31:3997-4013.

5. Dent R, Hanna WM, Trudeau M, et al. Pattern of metastatic spread in triple-negative breast cancer. Breast Cancer Res Treat 2009;115:423-428.

6. Dent R, Trudeau M, Pritchard KI, et al. Triple-negative breast cancer: clinical features and patterns of recurrence. Clin Cancer Res 2007;13(15 Pt 1):4429-4434.

7. Lin NU, Claus E, Sohl J, et al. Sites of distant recurrence and clinical outcomes in patients with metastatic triple-negative breast cancer: high incidence of central nervous system metastases. Cancer 2008;113:26382645.

8. Mazieres J, Peters S, Lepage B, et al. Lung cancer that harbors an HER2 mutation: epidemiologic characteristics and therapeutic perspectives. J Clin Oncol 2013;31:1997-2003.

9. Bose R, Kavuri SM, Searleman AC, et al. Activating HER2 mutations in HER2 gene amplification negative breast cancer. Cancer Discov 2013;3:224-237.

10. Anders CK, Carey LA. Biology, metastatic patterns, and treatment of patients with triple-negative breast cancer. Clin Breast Cancer 2009;9(Suppl 2):S73-81.

11. Su YC, Hsu YC, Chai CY. Role of TTF-1, CK20, and CK7 immunohistochemistry for diagnosis of primary and secondary lung adenocarcinoma. Kaohsiung J Med Sci 2006;22:14-19.

12. Dennis JL, Hvidsten TR, Wit EC, et al. Markers of adenocarcinoma characteristic of the site of origin: development of a diagnostic algorithm. Clin Cancer Res 2005;11:3766-3772.

13. Robens J, Goldstein L, Gown AM, Schnitt SJ. Thyroid transcription factor-1 expression in breast carcinomas. Am J Surg Pathol 2010;34:18811885 .
14. Erlander MG, Ma XJ, Kesty NC, et al. Performance and clinical evaluation of the 92-gene real-time PCR assay for tumor classification. J Mol Diagn 2011;13:493-503.

15. Varadhachary G. New strategies for carcinoma of unknown primary: the role of tissue-of-origin molecular profiling. Clin Cancer Res 2013;19:40274033.

16. Varadhachary GR, Raber MN. Cancer of unknown primary site. N Engl J Med 2014;371:757-765.

17. Ettinger DS, Handorf CR, Agulnik M, et al. Occult primary, version 3.2014. J Natl Compr Canc Netw 2014;12:969-974. To view the most recent version of these guidelines, visit NCCN.org.

18. Ganesan P, Moulder S, Lee JJ, et al. Triple-negative breast cancer patients treated at MD Anderson Cancer Center in phase I trials: improved outcomes with combination chemotherapy and targeted agents. Mol Cancer Ther 2014;13:3175-3184.

19. Tsimberidou AM, Iskander NG, Hong DS, et al. Personalized medicine in a phase I clinical trials program: the MD Anderson Cancer Center initiative. Clin Cancer Res 2012;18:6373-6383.

20. Weber JS, Levit LA, Adamson PC, et al. American Society of Clinical Oncology policy statement update: the critical role of phase I trials in cancer research and treatment. J Clin Oncol 2015;33:278-284.

21. Abrams J, Conley B, Mooney $M$, et al. National Cancer Institute's precision medicine initiatives for the new national clinical trials network. Am Soc Clin Oncol Educ Book 2014:71-76.

22. Fisher R, Pusztai L, Swanton C. Cancer heterogeneity: implications for targeted therapeutics. Br J Cancer 2013;108:479-485.

23. Yap TA, Gerlinger $M$, Futreal PA, et al. Intratumor heterogeneity: seeing the wood for the trees. Sci Transl Med 2012;4:127ps10.

24. Fabi A, Di Benedetto A, Metro G, et al. HER2 protein and gene variation between primary and metastatic breast cancer: significance and impact on patient care. Clin Cancer Res 2011;17:2055-2064.

25. Curtit E, Nerich V, Mansi L, et al. Discordances in estrogen receptor status, progesterone receptor status, and HER2 status between primary breast cancer and metastasis. Oncologist 2013;18:667-674.

26. Liedtke C, Broglio K, Moulder S, et al. Prognostic impact of discordance between triple-receptor measurements in primary and recurrent breast cancer. Ann Oncol 2009;20:1953-1958.

27. Phillips KA, Pletcher MJ, Ladabaum U. Is the "\$1000 Genome" really $\$ 1000$ ? Understanding the full benefits and costs of genomic sequencing [published online ahead of print February 6, 2015]. Technol Health Care, in press.

28. Weigelt $B$, Reis-Filho JS. Activating mutations in HER2: neu opportunities and neu challenges. Cancer Disc 2013;3:145-147.

29. Lee JW, Soung YH, Kim SY, et al. ERBB2 kinase domain mutation in the lung squamous cell carcinoma. Cancer Lett 2006;237:89-94.

30. Aertgeerts K, Skene R, Yano J, et al. Structural analysis of the mechanism of inhibition and allosteric activation of the kinase domain of HER2 protein. J Biol Chem 2011;286:18756-18765.

31. Stamos J, Sliwkowski MX, Eigenbrot C. Structure of the epidermal growth factor receptor kinase domain alone and in complex with a 4-anilinoquinazoline inhibitor. J Biol Chem 2002;277:46265-46272.

32. Baselga J, Cortes J, Kim SB, et al. Pertuzumab plus trastuzumab plus docetaxel for metastatic breast cancer. N Engl J Med 2012;366:109-119.

33. Pettersen EF, Goddard TD, Huang CC, et al. UCSF Chimera-a visualization system for exploratory research and analysis. J Comput Chem 2004;25:1605-1612.

34. Tamura K, Stecher G, Peterson D, et al. MEGA6: Molecular Evolutionary Genetics Analysis version 6.0. Mol Biol Evol 2013;30:2725-2729.

35. Hall TA. BioEdit: a user-friendly biological sequence alignment editor and analysis program for Windows 95/98/NT. Nucleic Acids Symp Ser 1999;41:95-98. 